\title{
P-0701 - High prevalence of peripheral neuropathy in type 2 diabetes: a hospital based cross sectional study from South India
}

\author{
P. Sankar ${ }^{1}$, G. Impana ${ }^{2}$, S. Kuppusamy ${ }^{3}$, R. Jacob ${ }^{1}$, S. Sasidharan ${ }^{1}$ \\ ${ }^{1}$ Diabetes Clinic, MGM Muthoot Medical Centre, Pathanamthitta- Kerala, India. \\ ${ }^{2}$ Apollo BGS Hospitals, Department of Family Medicine, Mysuru- Karnataka, India. \\ ${ }^{3}$ RIMS Hospital, Department of Family Medicine, Kottayam- Kerala, India.
}

\section{Background}

The burden of Type 2 Diabetes (T2DM) is rising equally in rural and urban India. Distal Peripheral Neuropathy (DPN) is the most prevalent chronic debilitating complication of T2DM. Loss of Protective sensation due to DPN is the main risk factor for amputation in patients with T2DM. Estimated prevalence of neuropathy has varied greatly in Indian studies (8\% to 59\%) depending on the method used, population screened and sample size. The state of Kerala in south India, despite having reported prevalence of T2DM as high as $20 \%$ (1), studies estimating prevalence of peripheral neuropathy in T2DM from the state are very few.

\section{Aim}

To establish the prevalence and associates of Distal Peripheral Neuropathy in patients with Type 2 Diabetes attending a multispecialty hospital in South Kerala.

\section{Method}

A cross-sectional study of 671 consecutive patients with T2DM was performed at the Outpatient Diabetes Clinic of a multispecialty hospital in Pathanamthitta district of South Kerala. Relevant history, clinical examination, laboratory tests were obtained from all patients. Patients with features suggestive of neuropathy due to alternate causes were excluded. Assessment for DPN was done by measuring Vibration Perception Threshold (VPT) using a biothesiometer, a well validated method. Peripheral Arterial Disease (PAD) was evaluated by measuring Ankle Brachial Index. Average VPT of more than $25 \mathrm{~V}$ measured by a single observer was defined as presence of DPN. Data collected based on a questionnaire was entered into Microsoft Excel. Statistical analysis including descriptive statistics and Chi- square test for categorical data was done using SPSSv15.

Results

Of the 671 patients, $40.1 \%$ were males. Mean age was $59.96 \pm 9.19$ years. $67.5 \%$ of patients had BMI more than $25 \mathrm{~kg} / \mathrm{m} 2$. Duration of T2DM was less than 10 years in $36.1 \%$. $40 \%$ had duration of diabetes more than 15 years. Mean $\mathrm{HbA1c}$ was $8.3 \pm 1.52 \%$. HbA1c was more than $8 \%$ in $54.7 \%$.

The overall prevalence of DPN in this population was $45.8 \%$. Evidence of Cardiovascular disease (CVD - Coronary Artery Disease, Stroke or TIA) was seen in $32.6 \%$. Prevalence of retinopathy was 25.5\%, increased Urine Albumin Excretion Rate (UAER) was $34.1 \%$ and PAD was $10.7 \%$. eGFR (CKD-EPI) was more than 60 $\mathrm{mL} / \mathrm{min} / 1.73 \mathrm{~m} 2$ in $80 \%$. Insulin was used by $48.6 \%$ of patients. $40.4 \%$ had their latest FBS less than $130 \mathrm{mg} / \mathrm{dL}$, while only $25.2 \%$ had their 2 hour PPBS below $180 \mathrm{mg} / \mathrm{dL} .81 .2 \%$ were on statins and $52.2 \%$ were on antiplatelets.
Presence of DPN was associated with increasing age ( $<<0.001) .17 .1 \%$ of patients below 50 years of age and $70.6 \%$ above 65 years had DPN. DPN was present in $27.3 \%$ patients less than 10 years duration and $56.2 \%$ of those having more than 15 years duration of T2DM $(p<0.001)$. Presence of DPN was strongly associated with CVD including PAD $(p<0.001)$, retinopathy $(p<0.001)$ and insulin use $(\mathrm{p}<0.001)$, after adjusting for age, duration of diabetes and HbA1c. In patients above 50 years of age presence of DPN was associated with increasing height of the individual $(\mathrm{p}<0.001)$. In patients with diabetes duration less than 10 years, DPN was associated with higher $\mathrm{HbA1c}$ levels $(\mathrm{P}=0.013)$. In patients with duration of diabetes more than 10 years, presence of DPN had significant associations with low eGFR $(p<0.001)$, increased UAER $(p<0.001)$, elevated systolic blood pressure $(\mathrm{p}=0.027)$. No significant association was observed with sex, BMI, smoking, dyslipidemia.

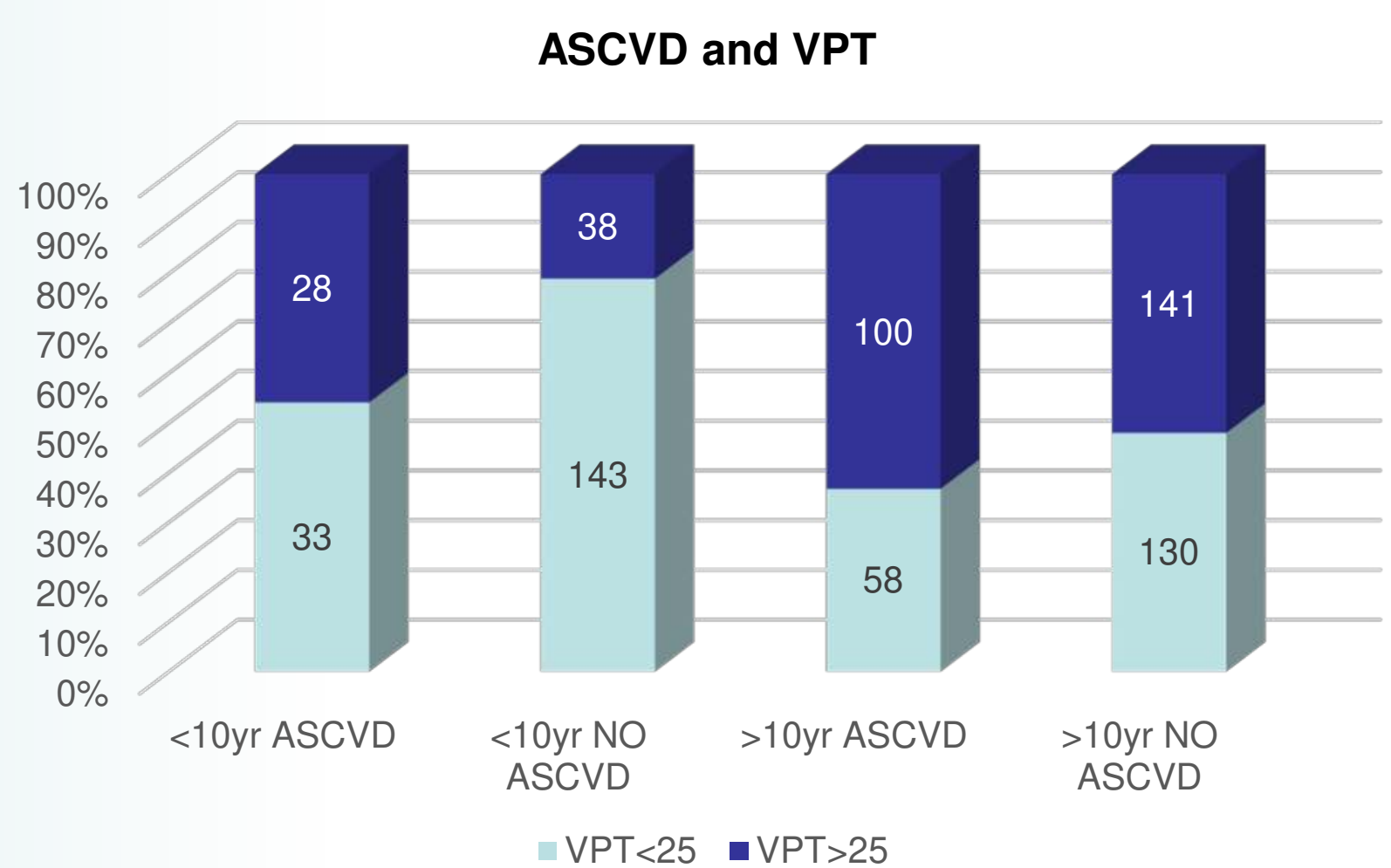

UAER, HEIGHT and VPT in >10yr Diabetes Duration

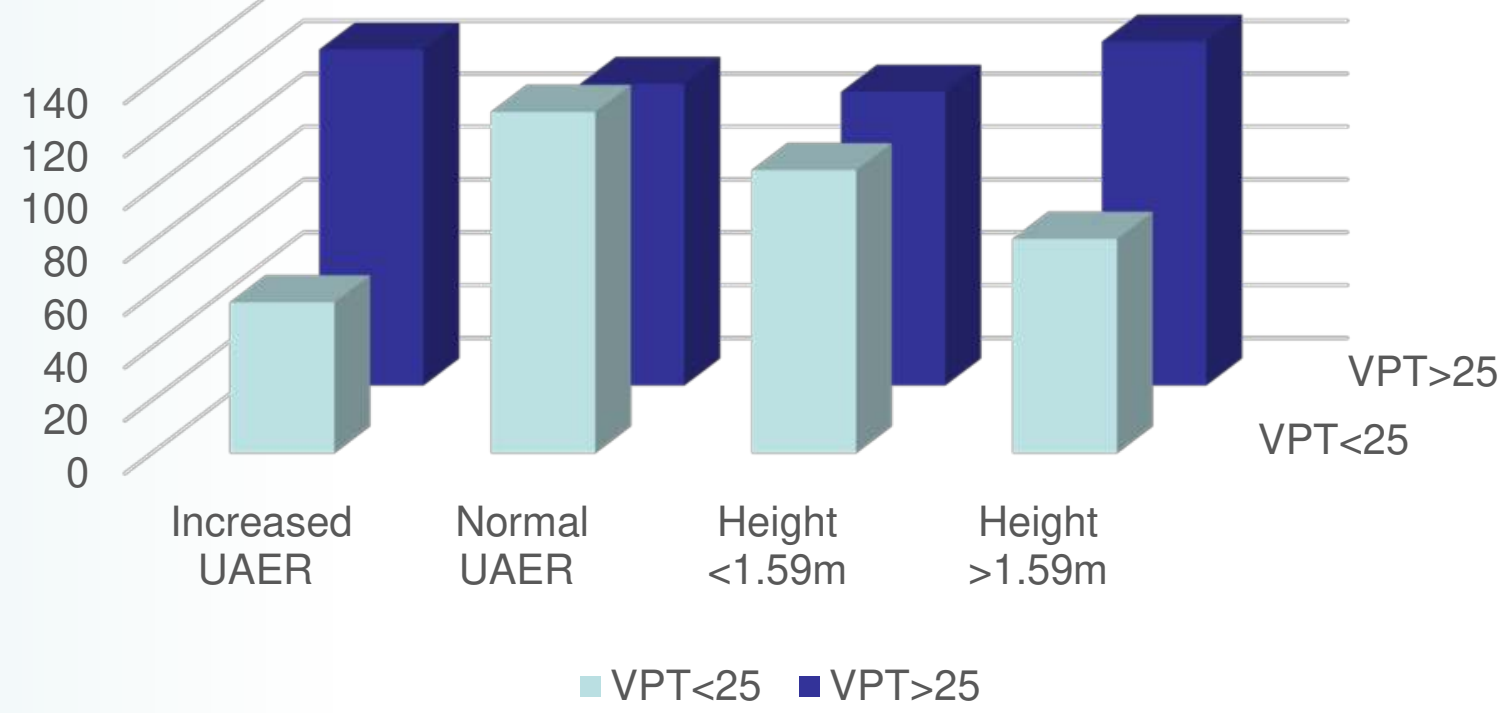

Discussion

The prevalence of DPN in patients with T2DM is very high in this population. To our knowledge, this is the first study from Kerala to estimate prevalence of DPN in T2DM. Our results are in concordance with similar studies from other regions of India and abroad. The study reaffirmed that poor glycemic control during early part of diabetes is a risk factor for DPN. The association between CVD and DPN shown by Brownrigg et al is observed in our study(2). Early onset of T2DM, improved healthcare delivery and increasing longevity in Kerala mean that the prevalence of DPN is rising. An increased awareness of the high prevalence of DPN, especially in older patients, should result in improved screening programs at primary care level in order to reduce the high incidence of neuropathic diabetic foot ulceration. 